\title{
Multiscale 3D Shape Analysis Using Spherical Wavelets
}

\author{
Delphine Nain ${ }^{1}$, Steven Haker ${ }^{2}$, Aaron Bobick ${ }^{1}$, and Allen R. Tannenbaum ${ }^{3}$ \\ 1 College of Computing, Georgia Institute of Technology, Atlanta, GA 30332-0280 \\ delfin@cc.gatech.edu, \\ 2 Department of Radiology, Surgical Planning Laboratory, \\ Brigham and Women's Hospital, Boston, MA 02115 \\ 3 Electrical Engineering, Georgia Institute of Technology, Atlanta, GA 30332-0250
}

\begin{abstract}
Shape priors attempt to represent biological variations within a population. When variations are global, Principal Component Analysis (PCA) can be used to learn major modes of variation, even from a limited training set. However, when significant local variations exist, PCA typically cannot represent such variations from a small training set. To address this issue, we present a novel algorithm that learns shape variations from data at multiple scales and locations using spherical wavelets and spectral graph partitioning. Our results show that when the training set is small, our algorithm significantly improves the approximation of shapes in a testing set over PCA, which tends to oversmooth data.
\end{abstract}

\section{Introduction}

Shape priors are commonly used to constrain shapes obtained during the segmentation and registration of biomedical images. Some of the first shape priors were based on local smoothness constraints [1] via elastic forces or combinations of global and local constraints 2 within the active contour framework. One limitation of these models is possible convergence to suboptimal shapes due to high flexibility in deformations. Statistical shape models were devised to overcome such drawbacks by learning a shape model from a training set. In 3 , PCA was used in a framework called Active Shape Models (ASM) and has become a standard technique for segmentation tasks [43]. The advantage of using PCA as a shape prior is to restrict the segmentation task to a subspace of allowable shapes. However, it has two major limitations. First, it often restricts deformable shape too much, particularly if it has been trained on a relatively small number of samples. Second, finer, more local variations of shapes are often not encoded in eigenvectors representing the most global modes of variation in the shapes.

To address this issue, the authors in [5] have proposed a hierarchical active shape model framework for contours in 2D medical imagery using wavelets, with convincing results. We propose to extend this framework in two novel ways. First we describe a multiscale representation of surfaces in 3D medical imagery using conformal mapping and spherical wavelets. Further, we present a novel algorithm to discover optimal independent multiscale shape variations from the data. Spherical wavelets have been used primarily by the computer graphics community to generate multiresolution description of 3D shapes [6]. In [7, spherical 
wavelets are used to analyze a manifold not topologically equivalent to a sphere by doing a non-bijective mapping between the manifold and the sphere via the normals. This work does not conduct statistical analysis of a shape population and uses a basis defined on the sphere, not on the shape.

To the best of our knowledge, this is the first application of statistical analysis to a population of 3D surfaces using spherical wavelets. By using a spherical wavelet basis defined on the shapes and identifying independent multiscale shape variations, we build more accurate shape priors.

\section{Shape Acquisition and Registration}

In this paper, we used a dataset of 39 prostate gland shapes obtained from MR imaging. In these images 1 the prostate capsule is visible and was manually segmented by a radiologist. Each manual segmentation defined a 3D surface which was extracted as a triangulated surface using the Marching Cubes algorithm. We registered all prostate shapes in the dataset by re-triangulating the extracted surfaces in a consistent manner, providing a point-by-point registration of all surfaces in the dataset. This re-triangulation was done by first mapping each surface to the sphere using a conformal (angle-preserving) mapping technique as described in [8. 6 Expert-specified landmark points were used to improve the consistency of the spherical mappings. Next, interpolation was used to find the coordinates of the original 3D surface at the vertices of a regular multiscale subdivision of the sphere, having an octahedral structure at its coarsest scale. Once corresponding points were identified, a Procrustes technique was used to align the shapes in the original coordinate system.

\section{Shape Representation}

Once registered, all shapes have $N$ vertices and each shape can be described by its three coordinate functions, $x, y, z \in \mathbb{R}$ such that the $k^{t h}$ shape $S_{k}$ is a column vector of size $3 N: S_{k}=\left[x_{k}(1), \ldots, x_{k}(N), y_{k}(1), \ldots, y_{k}(N), z_{k}(1), \ldots, z_{k}(N)\right]^{T}$.

Since all vertices in the shape population are registered, we interpret each entry of $S_{k}$ as a random variable and each shape as a realization from a multivariate probability distribution. A population of $K$ shapes can be described by a mean shape $\bar{S}=\frac{1}{K}\left(\sum_{k=1}^{K} S_{k}\right)$ and a set of transformations $T=\left[T_{1}, \ldots, T_{M}\right]$ that describe the variability observed in the population. Each transformation vector $T_{m}$ is of size $3 N$ where the $i^{t h}$ entry is a transformation applied to the $i^{\text {th }}$ entry of the mean shape with a corresponding magnitude $\beta_{m} \in \mathbb{R}$.

Each transformation vector, or variation mode, can be characterized by scale, spatial location and magnitude. For scale, the variation can be global, meaning it applies to all vertices of the shape (all of its entries are non-zero) or local, meaning it is a sparse vector with a few non-zero entries. The non-zero entries determine the spatial location of the variation. Characterization of local variations could be important for shape analysis since a disease, such as cancer, could affect only

\footnotetext{
${ }^{1}$ Axial, T2-weighted, $120 \mathrm{~mm}$ field of view, matrix size $256 \times 256,3.0 \mathrm{~mm}$ thickness, $0.5 \mathrm{~mm}$ gap, $1.5 T$, using both endorectal and pelvic coil arrays.
} 
a portion of an organ's surface. Therefore descriptive shape priors should discern shape variations at different scales and spatial location.

\section{Limitation of PCA in Representing Finer Shape Details}

Active shape models (ASM) 3] use principal component analysis (PCA) to discover uncorrelated shape variations from a training set. ASM assumes that training shapes have a multivariate normal distribution. The modes of variation are the eigenvectors of the data covariance matrix (major axes of the distribution).

If the training set is small, PCA favors discovery of significant global variations over local variations. Indeed, assuming a training set of $K$ shapes with $N$ vertices $(N \gg K)$, the rank of the covariance matrix, and number of eigenvectors, will be at most $K-1$. It can be shown that the eigenvectors associated with the largest eigenvalues of the covariance matrix describe the most significant modes of variation in the vertices [3]. This can be a feature of PCA, since it will enforce a global smoothness constraint on shapes, but also a limitation if shapes have important local variations, as will be shown in Section 6 .

\section{Multiscale Shape Prior Using Spherical Wavelets}

In this Section, we describe our technique to model the full range of variation in a population. We first derive a multiscale analysis of the variation of shapes in section 5.1. Section 5.2 details our shape prior for multiscale statistical shape analysis. Section 6 shows our results and compares our technique with ASM.

\subsection{Description of Spherical Wavelets}

To address this limitation, we propose a shape prior that represents variations at different scales and spatial locations. This can be achieved with wavelet basis functions that are localized in space and characteristic scales and therefore match a wide range of signal characteristics, from high frequency edges to slowly varying harmonics [9]. Spherical wavelets are second-generation wavelets adapted to manifolds with non-regular grids. We briefly sketch their construction [10].

Subdivision: Spherical wavelets analyze signals on a mesh obtained from recursive partitioning of a mesh with spherical topology. With the technique described in Section 2, all shapes have the required mesh to conduct the analysis. We denote the set of all vertices obtained after $j$ subdivisions with an index set $K(j)$. The $j+1^{t h}$ resolution mesh is obtained by introducing new nodes, identified by an index set $M(j)$ which subdivide existing edges (typically at their midpoint). The complete set of nodes in the $j+1^{t h}$ resolution mesh is given by $K(j+1)=K(j) \cup M(j)$ and shown in Figure 1(a) which represents a portion of a triangular surface mesh at resolution $j+1$.

Representation of shape functions: Let $S$ be a surface and let $\mathbf{x} \in \mathbb{R}^{3}$ be a point on $\mathrm{S}$. To approximate a function $\mathrm{f}(\mathbf{x})$ we use a set of wavelet and scaling basis functions defined on the shape and modulated by coefficients. For each resolution level, $\varphi_{j, .}(\mathbf{x})$ are hat-shaped scaling functions 2 defined at the nodes

\footnotetext{
${ }^{2}$ Varies linearly from value 1 at the vertex $x_{k}$ to 0 at neighboring vertices.
} 


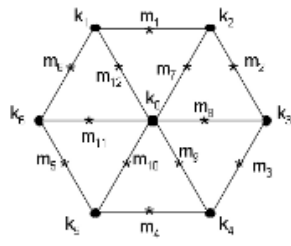

(a) grid

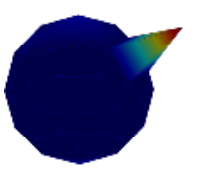

(b) $j=2$

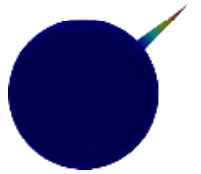

(c) $j=4$

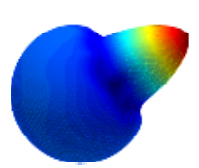

(d) $j=1$

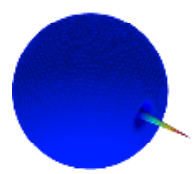

(e) $j=4$

Fig. 1. (a) Subdivision Grid. (b,c) Scaling (d,e) Wavelet Function for Different Nodes

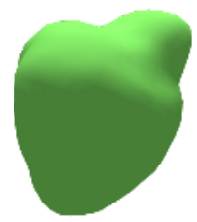

(a) shape

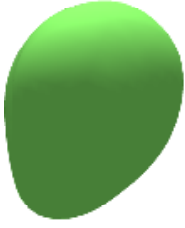

(b) res. 0

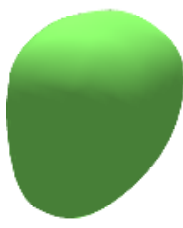

(c) res. 1

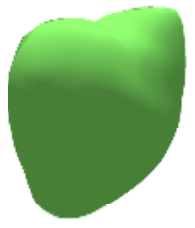

(d) res. 2

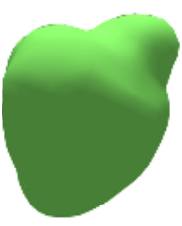

(e) res. 3

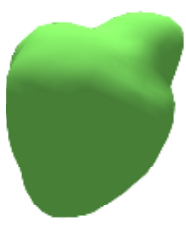

(f) res. 4

Fig. 2. Shape at various resolution levels, see text for details

$k \in K(j)$ and $\psi_{j, .}(\mathbf{x})$ are wavelet functions defined at nodes $m \in M(j)$. Wavelet functions capture finer features since they are composed of higher resolution $(j+1)$ scaling functions. Figures 1(b) - 1(e) show scaling and wavelet functions for different values of $j, k$ and $m$. Note that the support of the functions becomes smaller as the resolution increases.

When a shape is transformed into the wavelet domain, its wavelet coefficients are calculated 3 for all resolution levels:

$$
f(\mathbf{x})=\sum_{k \in K(0)} \lambda_{0, k} \varphi_{0, k}(\mathbf{x})+\sum_{0 \leq j} \sum_{m \in M(j)} \gamma_{j, m} \psi_{j, m}(\mathbf{x}) .
$$

A shape is represented by its lowest resolution scaling coefficients and its wavelet coefficients at all higher resolution levels. The total number of coefficients calculated by the transform is equal to the number of points representing the shape. Each coefficient describes features at a particular scale and spatial location.

Wavelet transform of the Prostate Data: We have applied the wavelet transform to prostate data to analyze multiscale variations in the population. We first subtract the mean shape from all the shapes in the set. We then apply the transform independently to the residual $x, y$ and $z$ coordinates of the $N$ vertices of the shape. The $k^{t h}$ shape in a population of $K$ shapes can be described by a vector of wavelet coefficients of size $3 N$ :

$$
\Gamma_{k}=\left\{\lambda_{0, k}^{x}, \lambda_{0, k}^{y}, \lambda_{0, k}^{z}, \gamma_{j, m}^{x}, \gamma_{j, m}^{y}, \gamma_{j, m}^{z} \mid j=0, . ., 4 ; m \in M(j) ; k \in K(0)\right\}
$$

Figure 2 shows a wavelet decomposition of a prostate. Figure 2(a) is the shape before decomposition. Each Figure 2(b) $2(\mathrm{f})$ is the mean shape plus the cumulative signals up to that resolution. We observe more high frequency content as the resolution increases.

\footnotetext{
${ }^{3}$ By inner product with dual functions, see [10] for more details.
} 


\subsection{Spherical Wavelets and Construction of Shape Priors}

The shape prior is the multivariate probability distribution of the coefficients, estimated from their covariance matrix $\Gamma \Gamma^{T}$. In our experiments, we have observed a very sparse covariance matrix with most of the dependency between coefficients at the same scale. Furthermore, for each scale we have observed clusters of correlated coefficients. This is consistent with the decorrelation property of the wavelet transform for real-world signals 9 . Given the sparseness of the covariance matrix, we approximate the joint distribution of coefficients with the product of distributions over smaller clusters of correlated coefficients.

Adaptive Band Selection by scale-space decomposition: For each scale, we cluster highly correlated coefficients into a band, with the constraint that coefficients across bands have minimum cross-correlation. Our technique is different from [5] where the authors perform a scale-space frequency decomposition of the coefficients by clustering coefficients of spatially adjacent bases into bands in each frequency plane. In this work, we cluster coefficients according to correlation to pick meaningful bands that indicate areas of variation. Such a decomposition can in itself be interesting for shape analysis.

To cluster correlated wavelet coefficients, we use a spectral graph partitioning technique [11. We use a fully connected undirected graph $G=(V, E)$ where nodes $V$ are wavelet coefficients for a particular scale. The weight on each edge $w(i, j)$ is the covariance between the wavelet coefficients at nodes $i$ and $j$. A $\operatorname{cut}(A, B)=\sum_{u \in A, v \in B} w(u, v)$ is the optimal partitioning of $\mathrm{V}$ into two disjoint sets $A$ and $B$ such that nodes within a partition have the highest covariance and nodes across partitions have the lowest covariance.

Using this technique, we do a recursive partitioning of the coefficients into bands. We use a stopping criteria based on the quality of the decomposition of each set, validating whether the subdivided band correspond to two independent distributions. Indeed, if we start with a graph $G$ where we consider each coefficient to be a random variable and find a partition $A \cup B=G, A \cap B=\emptyset$, then

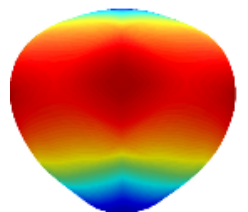

(a) $B_{0,1}, \mathrm{~A}$

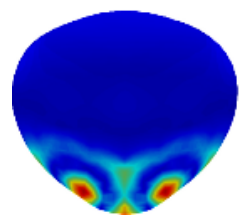

(e) $B_{(2,2)}, \mathrm{A}$

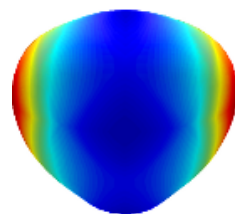

(b) $B_{0,1}, \mathrm{P}$

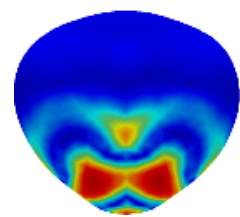

(f) $B_{2,2}, \mathrm{P}$

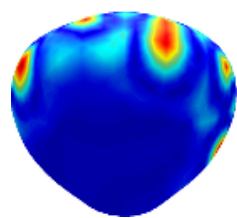

(c) $B_{2,1} \mathrm{~A}$

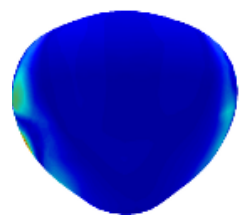

(g) $B_{2,3}, \mathrm{~A}$

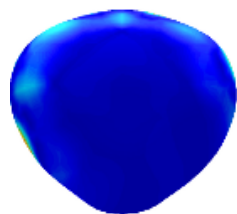

(d) $B_{2,1}, \mathrm{P}$

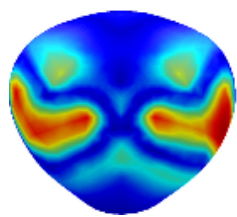

(h) $B_{2,3}, \mathrm{P}$

Fig. 3. Band Decomposition: various bands $B_{j, i}$, where $j$ is the resolution and $i$ is the band number, shown in Anterior view (A) and Posterior view (P), see text for color 
it is a good partition if $P(G)=P(A) P(B)$. We can test this with the KullbackLeibler (KL) divergence between the joint and the product of the marginals.

In this work, we assume a multivariate Gaussian distribution for each partition and derived the KL divergence [12]:

$$
D(P(G)|| P(A) P(B))=1 / 2 \log \left(\left|\Sigma_{A}\right|\left|\Sigma_{B}\right|\right)-1 / 2 \log \left(\left|\Sigma_{G}\right|\right)
$$

where $\Sigma_{A}$ is the covariance matrix of $P(A)$ and $|$.$| is the determinant 4$. If the distributions $P(A)$ and $P(B)$ are independent, then their KL divergence is 0 . In practice, we do not accept a partition if $D(P(G) \| P(A) P(B))>0.1$.

Band Visualization: To visualize the bands, we calculate the influence of all wavelet coefficient in band $B_{j, i}$ on each point $\mathbf{x}$ of the surface by setting those coefficients to 1 in (11) and others to 0 . If $f(\mathbf{x})=0$, then the point is not affected and if $f(\mathbf{x})>0$ it is affected according to the magnitude of $f(\mathbf{x})$. Using this function as a colormap (blue $=0$, red $=1$ ), Figures $3(\mathrm{a})-3(\mathrm{~b})$ show the first band for the lowest scale. The second band is the complement of the first. As expected each band has a large spatial extent and indicate two uncorrelated shape processes on the prostate data: the variation of the anterior wall of the prostate (typically rounded) and the variation of the posterior wall of the prostate (typically flatter). Figures $3(\mathrm{c})-3(\mathrm{~h})$ show three bands for the scale 3 . These bands are more localized. These are uncorrelated variations of the superior and inferior walls of the shape, as well as an uncorrelated variations of the anterior wall at that scale. Bands have compact support, though this is not a constraint of our technique. The symmetry in bands 2 and 3 is also interesting, showing that both the right and left side tend to co-vary similarly. This symmetry of variation is plausible for the prostate, and we plan to investigate this further. Notably a diseased organ could possibly be detected if there is a lack of symmetry.

Building the Prior: Each band of coefficients is modeled as a multivariate Gaussian that encodes a set of shape variations. To estimate their distribution from the data, we apply PCA analysis to the coefficients in each band. The eigenvectors and eigenvalues of lower scale bands represent relatively global aspects of shape variability, whereas bands at higher scales represent higher frequency and more localized aspects of shape variability. Hence, our technique discovers shape processes at every scale, where the processes are all the eigenvectors of all the bands, and does not favor the discovery of global processes over local processes. Additionally, our prior accurately encodes finer details even with small training sets, since there exists at least $B(K-1)$ eigenvectors for each scale with $B$ bands.

\section{Experiments}

We compare our technique to PCA for the task of reconstruction. We partition our data randomly into $\mathrm{T}$ training samples and $39-T$ testing samples, where $T=[5,10,20,30]$ and learn a shape prior from the training set. The prior for

${ }^{4}$ In practice since $\Sigma_{A}$ is often singular, we decompose it using SVD as $\Sigma_{A}=U \Sigma_{A}^{\prime} U^{T}$ and estimate $\log \left|\Sigma_{A}\right|=\operatorname{trace}\left(\log \left(\Sigma_{A}^{\prime}\right)\right)$, using only the positive entries of $\Sigma_{A}^{\prime}$. 
PCA consists of the mean shape, the eigenvectors and eigenvalues. The prior for our technique consists of the mean shape, the bands structure, the eigenvectors and eigenvalues for each band. We then reconstruct shapes in the testing set.

For PCA, we project the training shape coordinates onto the eigenvectors and translate the coordinates of the new shape to a point lying at a reasonable distance of the training data ( \pm 3 standard deviation) to obtain a reconstructed shape. For our technique, we transform the shape to its wavelet coefficients, project and correct the coefficients for each band separately, and transform the new coefficients back to a reconstructed shape. A mean squared error between the vertices of the ground truth and the reconstructed shape is calculated for all shapes in the testing set.

Figure 4 shows the reconstruction error with the multiscale prior (solid line). It is significantly smaller than for PCA (dashed line) across all training set sizes.

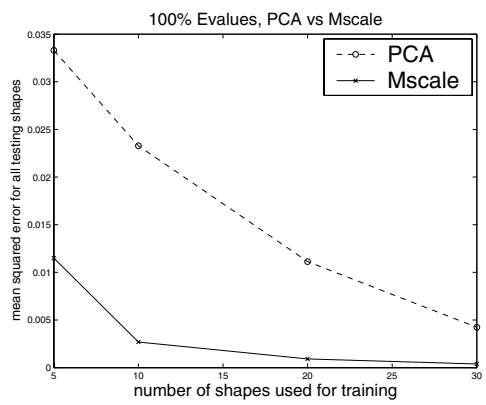

Fig. 4. Mean Squared Reconstruction Error for various training set sizes

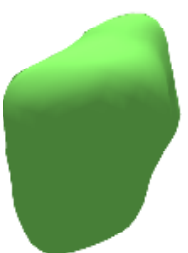

(a) GT

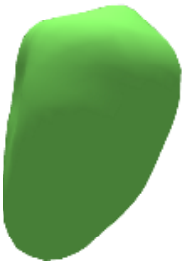

(g) GT

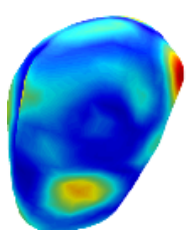

(b) PCA

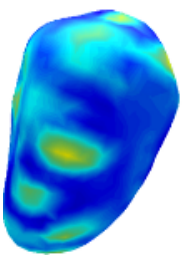

(h) PCA

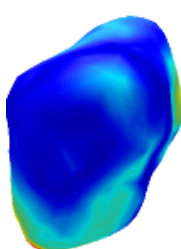

(c) Mscale

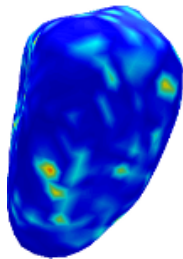

(i) Mscale

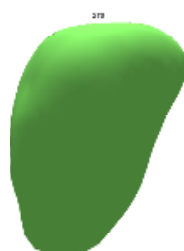

(d) GT

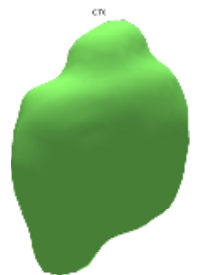

(j) GT

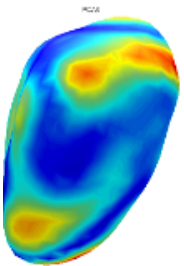

(e) PCA

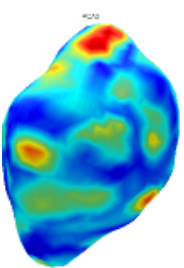

(k) PCA

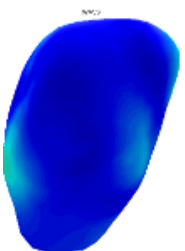

(f) Mscale

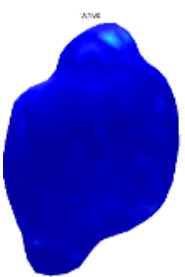

(1) Mscale

Fig. 5. Ground Truth (GT) and reconstruction with PCA and Multiscale priors (a-f) 5 training samples (g-l) 30 training samples. Color is error from blue (lowest) to red. 
The error for PCA significantly increases when the training set size decreases, but only slightly increases for the multiscale technique.

Figures $5(\mathrm{a}), 5(\mathrm{~d})$, show a ground truth test shape and $5(\mathrm{~b}), 5(\mathrm{e}), 5(\mathrm{c}), 5(\mathrm{f})$ its reconstruction with the PCA and multiscale shape priors built from 5 training shapes. For PCA, the reconstruction is very smooth. Many of the finer details are lacking in the reconstruction. The multiscale technique incorporates both local and global details that PCA does not encode. Figures $5(\mathrm{~g}), 5(\mathrm{j})$ show a ground truth test shape and $5(\mathrm{~h}), 5(\mathrm{k}), 5(\mathrm{i}), 5(\mathrm{l})$ its reconstruction from 30 training shapes. For PCA, the reconstruction is more accurate, but still lacks some finer details, such as the sharper edges. Again, the multiscale technique incorporates both local and global details that PCA misses.

\section{Conclusions and Future Work}

We have demonstrated that our spherical wavelet based technique is a better shape prior than ordinary PCA when it is important to represent finer, more localized shape variation. Our novel method finds independent shape variation processes at multiple scales and multiple locations by adaptively clustering correlated wavelet coefficients. The visualization of the bands can, in itself, be an interesting tool for shape analysis. We plan in future work to compare this choice of $L^{2}$ basis to other natural bases such as spherical harmonics 13 , as well as investigate the use of wavelet packets [14]. We also plan to use it for key imaging tasks such as segmentation. Further, we intend to build on this theory in order to derive a natural multiscale description for discriminative shape analysis. Localized processes cannot be overlooked when discriminating among populations, making this technique promising for localized lesions, such as tumors. Finally, we will be applying this methodology to other types of structures, in particular, to the caudate nucleus as part of our research in schizophrenia.

Acknowledgements. This work is part of the National Alliance for Medical Image Computing (NAMIC), funded through the NIH Roadmap for Medical Research, Grant U54 EB005149. A. Tannenbaum and D. Nain's work was also funded by NIH NAC grant P41 RR-13218 and S. Haker's work is supported by NIH grants R01CA109246, R01LM007861, R01CA1029246 and P41RR019703.

\section{References}

1. Kass, M., Witkin, A., Terzopoulos, D.: Snakes: Active contour models. Int. J. Comput. Vis 1 (1987) 321-331

2. Terzopoulos, D., Metaxas, D.: Dunamic 3d models with local and global deformations: Deformable superquadrics. IEEE Trans. Pat. Anal. Mach. Intell. 13 (1991)

3. Cootes, T., Taylor, C., Cooper, D., Graham, J.: Active shape models - their training and application. Computer Vision and Image Understanding 61 (1995) 38-59

4. Leventon, M., Grimson, E., Faugeras, O.: Statistical shape influence in geodesic active contours. Comp. Vision and Patt. Recon. (CVPR) (2000)

5. Davatzikos, C., Tao, X., Shen, D.: Hierarchical active shape models, using the wavelet transform. IEEE Trans. Medical Imaging 22 (2003) 414-423 
6. Schröder, P., Sweldens, W.: Spherical wavelets: Efficiently representing functions on the sphere. Computer Graphics Proceedings (SIGGRAPH 95) (1995) 161-172

7. Greenshields, I.: 3d shape approximants via spherical wavelet decompositions. 14th Symposium on Computer-Based Medical Systems (2001) 31

8. Haker, S., Warfield, S., Tempany, C.: Landmark-guided surface matching and volumetric warping for improved prostate biopsy targeting. MICCAI (2004)

9. Mallat, S.: Wavelet Tour of Signal Processing. Academic Press (1999)

10. Schröder, P., Sweldens, W.: Spherical wavelets: Texture processing. In Hanrahan, P., Purgathofer, W., eds.: Rendering Techniques '95. Springer Verlag (1995)

11. Shi, J., Malik, J.: Normalized cuts and image segmentation. IEEE Transactions on Pattern Analysis and Machine Intelligence 22 (2000) 888-905

12. Papoulis, A.: Probability, Random Variables, and Stochastic Processes. McGrawHill (1991)

13. Styner, M., Lieberman, J.A., Pantazis, D., Gerig, G.: Boundary and medial shape analysis of the hippocampus in schizophrenia. Medical Image Analysis 8 (2004)

14. Mohamed, A., Davatzikos, C.: Shape representation via best orthogonal basis selection. MICCAI 1 (2004) 225-233 